\title{
Piecewise-linear and birational toggling
}

\author{
David Einstein* and James Propp ${ }^{\dagger}$ \\ Department of Mathematical Sciences, UMass Lowell, USA
}

\begin{abstract}
We define piecewise-linear and birational analogues of toggle-involutions, rowmotion, and promotion on order ideals of a poset $P$ as studied by Striker and Williams. Piecewise-linear rowmotion relates to Stanley's transfer map for order polytopes; piecewise-linear promotion relates to Schützenberger promotion for semistandard Young tableaux. When $P=[a] \times[b]$, a reciprocal symmetry property recently proved by Grinberg and Roby implies that birational rowmotion (and consequently piecewise-linear rowmotion) is of order $a+b$. We prove some homomesy results, showing that for certain functions $f$, the average of $f$ over each rowmotion/promotion orbit is independent of the orbit chosen.

Résumé. Nous définissons et étudions certains analogues linéaires-par-morceaux et birationnels d'involutions toggles, rowmotion et promotion sur les idéaux d'un poset $P$, comme étudié par Striker et Williams. La rowmotion linéairepar-morceaux est liée à la fonction transfert de Stanley pour les polytopes d'ordre; la promotion linéaire-par-morceaux se rapporte à la promotion de Schützenberger pour les tableaux semistandards de Young. Lorsque $P=[a] \times[b]$, une propriété de symétrie réciproque récemment prouvée par Grinberg et Roby implique que la rowmotion birationnelle (et par conséquent la rowmotion linéaire-par-morceaux) est de l'ordre $a+b$. Nous démontrons quelques résultats d'homomesie, montrant que pour certaines fonctions $f$, la moyenne de $f$ sur chaque orbite de rowmotion/promotion est indépendante de l'orbite choisie.
\end{abstract}

Keywords: poset, order ideal, order polytope, rowmotion, promotion, tropicalization

\section{Background}

We assume readers are familiar with the definition of a finite poset $(P, \leq)$, as for instance given in Ch. 3 of Stanley (2011). Much of our work involves the case $P=[a] \times[b]=\{(i, j) \in \mathbb{N} \times \mathbb{N}: 1 \leq i \leq a, 1 \leq$ $j \leq b\}$ with ordering defined by $(i, j) \leq\left(i^{\prime}, j^{\prime}\right)$ iff $i \leq i^{\prime}$ and $j \leq j^{\prime}$. We put $n=a+b$.

We write $x \lessdot y$ (" $x$ is covered by $y$ ") or equivalently $y>x$ (" $y$ covers $x$ ") when $x<y$ and no $z \in P$ satisfies $x<z<y$. We say $P$ is ranked if there is a function $r: P \rightarrow\{0,1,2, \ldots\}$ so that all minimal elements of $P$ have rank 0 and such that $x \lessdot y$ implies $r(x)=r(y)-1$.

An $r$-embedding of a poset $P$ is defined by Striker and Williams (2012) as a map $\pi: P \rightarrow \mathbb{Z} \times \mathbb{Z}$ such that $x$ covers $y$ iff $\pi(x)-\pi(y)$ is $(1,1)$ or $(-1,1)$. This yields a Hasse diagram for $P$ in which all covering relations are edges of slope \pm 1 . In the case $P=[a] \times[b]$, we will adopt the rc-embedding $\pi$ that sends $(i, j) \in P$ to $(j-i, i+j-2) \in \mathbb{Z}^{2}$. The ranks (or, in the terminology of Striker and Williams

\footnotetext{
*Email: deinstegmail.com.

${ }^{\dagger}$ Homepage: http: / / jamespropp.org.
} 
(2012), rows) are the subsets of $P$ that consist of all $x \in P$ at a given height, or vertical position, relative to the rc-embedding. We define the files (or, in the terminology of Striker and Williams (2012), columns) as the subsets of $P$ that consist of all $x \in P$ at a given horizontal position relative to the rc-embedding. For example, let $P=[2] \times[2]$, and write $(1,1),(2,1),(1,2),(2,2)$ as $w, x, y, z$ for short, with $w<x<z$ and $w<y<z$. Our rc-embedding has $\pi(w)=(0,0), \pi(x)=(-1,1), \pi(y)=(1,1)$, and $\pi(z)=(0,2)$.

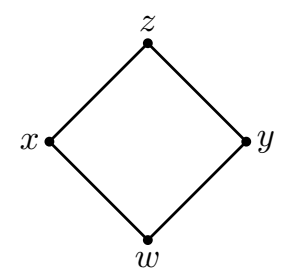

The ranks are $\{w\},\{x, y\}$, and $\{z\}$, and the files are $\{x\},\{w, z\}$, and $\{y\}$. We number the ranks of $[a] \times[b]$ from 0 (bottom) to $n-1$ (top), and we number the files of $[a] \times[b]$ from 1 (left) to $n$ (right). That is, for $P=[a] \times[b],(i, j) \in P$ belongs to the $(i+j-2)$ nd rank of $P$ and the $(j-i+a) \operatorname{th}^{(\mathrm{i})}$ file of $P$.

We call $S \subseteq P$ an order ideal (or downset) of $P$ when $x \in S$ and $y \leq x$ imply $y \in S$. We call $S \subseteq P$ a filter (or upset) of $P$ when $x \in S$ and $y \geq x$ imply $y \in S$. We call $S \subseteq P$ an antichain when $x, y \in S$ and $x \neq y$ imply that $x$ and $y$ are incomparable (i.e., neither $x \leq y$ nor $y \leq x$ ). The sets consisting of the order ideals, filters, and antichains of $P$ are respectively denoted by $J(P), \mathcal{F}(P)$, and $\mathcal{A}(P)$.

There are natural bijections $\alpha_{1}: J(P) \rightarrow \mathcal{F}(P), \alpha_{2}: \mathcal{F}(P) \rightarrow \mathcal{A}(P)$, and $\alpha_{3}: \mathcal{A}(P) \rightarrow J(P)$ given by the following recipes:

(1) for $I \in J(P)$, let $\alpha_{1}(I)$ be the complement $P \backslash I$;

(2) for $F \in \mathcal{F}(P)$, let $\alpha_{2}(F)$ be the set of minimal elements of $F$ (i.e., the set of $x \in F$ such that $y<x$ implies $y \notin F)$; and

(3) for $A \in \mathcal{A}(P)$, let $\alpha_{3}(A)$ be the downward saturation of $A$ (i.e., the set of $y \in P$ such that $y \leq x$ for some $x \in A$ ).

The composition $\rho:=\alpha_{3} \circ \alpha_{2} \circ \alpha_{1}: J(P) \rightarrow J(P)$ is not the identity map; e.g., it sends the full order ideal $I=P$ to the empty order ideal $I=\emptyset$. (Note that Brouwer and Schrijver (1974) studied the closely related map $F=\alpha_{2} \circ \alpha_{1} \circ \alpha_{3}: \mathcal{A}(P) \rightarrow \mathcal{A}(P)$.)

Cameron and Fon-Der-Flaass (1995) gave an alternative characterization of $\rho$. Given $x \in P$ and $I \in J(P)$, let $\tau_{x}(I)$ (" $I$ toggled at $x$ " in Striker and Williams' terminology) denote the set $I \triangle\{x\}$ if this set is in $J(P)$ and $I$ otherwise. Equivalently, $\tau_{x}(I)$ is $I$ unless $y \in I$ for all $y \lessdot x$ and $y \notin I$ for all $y \gg x$, in which case $\tau_{x}(I)$ is $I \triangle\{x\}$. (We will sometimes say that "toggling $x$ turns $I$ into $\tau_{x}(I)$ ".) Clearly $\tau_{x}$ is an involution. It is easy to show that $\tau_{x}$ and $\tau_{y}$ commute unless $x \lessdot y$ or $x \gg y$. If $x_{1}, x_{2}, \ldots, x_{|P|}$ is any linear extension of $P$ (that is, a listing of the elements of $P$ such that $x_{i}<x_{j}$ in $P$ implies $i<j$ in $\mathbb{N}$ ), then the composition $\tau_{x_{1}} \circ \tau_{x_{2}} \circ \cdots \circ \tau_{x_{|P|}}$ coincides with $\rho$. In the case where the poset $P$ is ranked, one natural way to linearly extend $P$ is to list the elements in order of increasing rank. Given the right-to-left order of composition of $\tau_{x_{1}} \circ \tau_{x_{2}} \circ \cdots \circ \tau_{x_{|P|}}$, this corresponds to toggling the top rank first, then the next-to-top rank, and so on, lastly toggling the bottom rank. When $x$ and $y$ belong to the same rank of $P$, the toggle operations $\tau_{x}$ and $\tau_{y}$ commute, so even without using the theorem of Cameron and

(i) Note that $j-i+a$ ranges from 1 to $a+b-1=n-1$; this is slightly different from the indexing in Propp and Roby (2013). 
Fon-der-Flaass, we can see that this composite operation on $J(P)$ is well-defined. Striker and Williams (2012) use the term "row" as a synonym for "rank", and they refer to $\rho$ as rowmotion.

In the example above, under the action of $\alpha_{1}, \alpha_{2}$, and $\alpha_{3}$, the order ideal $\{w, x\} \in J(P)$ gets successively mapped to $\{y, z\} \in \mathcal{F}(P),\{y\} \in \mathcal{A}(P)$, and $\{w, y\} \in J(P)$. Under the action of $\tau_{z}, \tau_{y}, \tau_{x}$, and $\tau_{w}$, the order ideal $\{w, x\} \in J(P)$ gets successively mapped to $\{w, x\},\{w, x, y\},\{w, y\}$, and $\{w, y\}$ (all in $J(P))$. In both cases we obtain $\rho(\{w, x\})=\{w, y\}$.

Note that if $x$ and $y$ belong to the same file, the toggle operations $\tau_{x}$ and $\tau_{y}$ commute, since neither of $x, y$ can cover the other. Thus the composite operation of toggling the elements of $P$ from left to right is well-defined; Striker and Williams (2012) call this operation promotion, and show that it is conjugate to rowmotion in the toggle group (the group generated by the toggle involutions). We denote this map by $\pi$.

\section{Piecewise-linear toggling}

Given a poset $P=\left\{x_{1}, \ldots, x_{p}\right\}$ (with $p=|P|$ ) and an rc-embedding of $P$, let $\mathbb{R}^{P}$ denote the set of functions $f: P \rightarrow \mathbb{R}$; we can represent such an $f$ as a $P$-array (or array for short) in which the values of $f(x)$ for all $x \in P$ are arranged on the page according to the rc-embedding of $P$ in the plane. We will sometimes identify $\mathbb{R}^{P}$ with $\mathbb{R}^{p}$, associating $f \in \mathbb{R}^{P}$ with $v=\left(f\left(x_{1}\right), \ldots, f\left(x_{p}\right)\right)$, though this depends on the chosen ordering of the elements of $P$. Let $\widehat{P}$ denote the augmented poset obtained from $P$ by adding two extra elements $\widehat{0}$ and $\widehat{1}$ (which we sometimes denote by $x_{0}$ and $x_{p+1}$ ) satisfying $\widehat{0}<x<\widehat{1}$ for all $x \in P$. The order polytope $\mathcal{O}(P) \subset \mathbb{R}^{p}$ (see Stanley (1986)) is the set of vectors $\left(\widehat{f}\left(x_{1}\right), \ldots, \widehat{f}\left(x_{p}\right)\right)$ in $\mathbb{R}^{p}$ arising from functions $\widehat{f}: \widehat{P} \rightarrow \mathbb{R}$ that satisfy $\widehat{f}(\widehat{0})=0$ and $\widehat{f}(\widehat{1})=1$ and are order-preserving ( $x \leq y$ in $P$ implies $\widehat{f}(x) \leq \widehat{f}(y)$ in $\mathbb{R}$ ). In some cases it is better to work with the augmented vector $\left(\widehat{f}\left(x_{0}\right), \widehat{f}\left(x_{1}\right), \ldots, \widehat{f}\left(x_{p}\right), \widehat{f}\left(x_{p+1}\right)\right)$ in $\mathbb{R}^{p+2}$. In either case we have a convex compact polytope.

For example, if $P=[2] \times[2]=\{w, x, y, z\}$, then $\mathcal{O}(P)=\left\{v=\left(v_{1}, v_{2}, v_{3}, v_{4}\right) \in \mathbb{R}^{4}: 0 \leq v_{1}\right.$, $v_{1} \leq v_{2}, v_{1} \leq v_{3}, v_{2} \leq v_{4}, v_{3} \leq v_{4}$, and $\left.v_{4} \leq 1\right\}$; each such $v$ can be depicted as the $P$-array

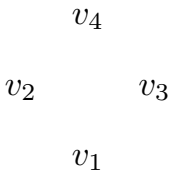

$\mathcal{O}(P)$ is the convex hull of the vectors $(0,0,0,0),(0,0,0,1),(0,0,1,1),(0,1,0,1),(0,1,1,1)$, and $(1,1,1,1)$, which are precisely the vectors associated with the filters of $P$. It is shown in Stanley (1986) that for any poset $P$, the vertices of $\mathcal{O}(P)$ correspond to the indicator functions of the filters of $P$.

Given a convex compact polytope $K$ in $\mathbb{R}^{p}$ (we are only concerned with the case $K=\mathcal{O}(P$ ) here but the definition makes sense more generally), we define the piecewise-linear toggle operation $\tau_{i}(1 \leq i \leq p)$ as the unique map from $K$ to itself whose action on the 1-dimensional cross-sections of $K$ in the $i$ th coordinate direction is the linear map that switches the two endpoints of the cross-section. That is, given $v=\left(v_{1}, \ldots, v_{p}\right) \in K$, we define

$$
\tau_{i}(v)=\left(v_{1}, \ldots, v_{i-1}, L+R-v_{i}, v_{i+1}, \ldots, v_{p}\right)
$$

where the real numbers $L$ and $R$ are respectively the left and right endpoints of the set $\{t \in \mathbb{R}:$ 
$\left.\left(v_{1}, \ldots, v_{i-1}, t, v_{i+1}, \ldots, v_{p}\right) \in K\right\}$, which is a bounded interval because $K$ is convex and compact. (ii) Since $L+R-\left(L+R-v_{i}\right)=v_{i}$, each toggle operation is an involution.

Similar involutions were studied by Kirillov and Berenstein (1995) in the context of Gelfand-Tsetlin triangles. Indeed, one can view their action in our piecewise-linear toggling framework, where instead of looking at the rectangle posets $[a] \times[b]$ one looks at the triangle posets with elements $\{(i, j): 1 \leq i \leq$ $j \leq N\}$ and covering-relations $(i, j-1) \lessdot(i, j)$ (for $1 \leq i \leq j \leq N)$ and $(i+1, j+1) \lessdot(i, j)$ (for $1 \leq i \leq j \leq N-1$ ). Their "elementary transformations" (Definition 0.1) are our "toggles".

In the case where $K$ is the order polytope of $P$ and a particular element $x \in P$ has been indexed as $x_{i}$, we write $\tau_{i}$ as $\tau_{x}$. The $L$ and $R$ that appear in (1) are given by

$$
L=\max \left\{v_{j}: 0 \leq j \leq p+1, x_{j} \lessdot x_{i}\right\}
$$

and

$$
R=\min \left\{v_{j}: 0 \leq j \leq p+1, x_{j} \gg x_{i}\right\} .
$$

(One also has $L=\max \left\{v_{j}: x_{j}<x_{i}\right\}$ and $R=\min \left\{v_{j}: x_{j}>x_{i}\right\}$, but the formulas (1)-(3) turn out to be the right ones to use when extending the operations $\tau_{i}$ from $\mathcal{O}(P)$ to all of $\mathbb{R}^{p}$, as well as the right ones to use when lifting toggling to the birational setting as described in the next section.) It is easy to show that $\tau_{x}$ and $\tau_{y}$ commute unless $x \lessdot y$ or $x>y$. These piecewise-linear toggle operations $\tau_{x}$ are analogous to the combinatorial toggle operations $\tau_{x}$ (and indeed the former generalize the latter in a sense to be made precise below), so it is natural to define piecewise-linear rowmotion $\rho_{\mathcal{P}}: \mathcal{O}(P) \rightarrow \mathcal{O}(P)$ as the composite operation accomplished by toggling from top to bottom (much as ordinary rowmotion $\rho: J(P) \rightarrow J(P)$ can be defined as the composite operation obtained by toggling from top to bottom). Likewise we can define piecewise-linear promotion $\pi_{\mathcal{P}}: \mathcal{O}(P) \rightarrow \mathcal{O}(P)$ as the composite operation accomplished by toggling from left to right.

Continuing the example $P=[2] \times[2]=\{w, x, y, z\}$ from section 1 , let $v=(.1, .2, .3, .4) \in \mathcal{O}(P)$. Under the action of $\tau_{z}, \tau_{y}, \tau_{x}$, and $\tau_{w}$, the vector $v$ gets successively mapped to $(.1, .2, .3, .9),(.1, .2, .7, .9)$, $(.1, .8, .7, .9)$, and $(.6, .8, .7, .9)=\rho_{\mathcal{P}}(v)$, while under the action of $\tau_{x}, \tau_{w}, \tau_{z}$, and $\tau_{y}$, the vector $v$ gets successively mapped to $(.1, .3, .3, .4),(.2, .3, .3, .4),(.2, .3, .3, .9)$, and $(.2, .3, .8, .9)=\pi_{\mathcal{P}}(v)$.

If $f$ is the indicator function of the filter $P \backslash I$, then $\rho_{\mathcal{P}}(v)$ (resp. $\pi_{\mathcal{P}}(v)$ ) is the indicator function of the filter $P \backslash \rho(I)$ (resp. $P \backslash \pi(I)$ ); in this way $\rho_{\mathcal{P}}$ and $\pi_{\mathcal{P}}$ generalize $\rho$ and $\pi$.

In the full version of the article (Einstein and Propp (2014)), we extend $\rho_{\mathcal{P}}$ and $\pi_{\mathcal{P}}$ to all of $\mathbb{R}^{p}$, not just $\mathcal{O}(P)$. We also study a variant of these extended operations in which one takes $(\widehat{f}(\widehat{0}), \widehat{f}(\widehat{1}))=(0,0)$ instead of $(0,1)$; although there is no longer an order polytope in the picture, these "homogeneous" actions are easier to understand, and capture most of the behavior of the general inhomogeneous case.

One can show that the action of the Schützenberger promotion operator (which we denote by $\pi_{\mathcal{S}}$ ) on the set of semistandard Young tableaux of rectangular shape with $A$ rows and $B$ columns having entries between 1 and $n$ is naturally conjugate to the action of the piecewise-linear promotion operator $\pi_{\mathcal{P}}$ on the rational points in the order polytope of $P=[A] \times[n-A]$ with denominator dividing $B$. (We are grateful to Alex Postnikov and Darij Grinberg for explaining this to us. For the original definition of promotion, see Schützenberger (1972); for more modern treatments, see Stanley (2009) and van Leeuwen (1996).) For example, take $A=2, B=3$, and $n=5$, and consider the semistandard Young tableau

$$
\begin{array}{lll}
1 & 2 & 2 \\
3 & 5 & 5
\end{array}
$$

(ii) Note that $L$ and $R$ depend on $v_{1}, \ldots, v_{i-1}, v_{i+1}, \ldots, v_{p}$, though our notation suppresses this dependence. 
We represent the tableau $T$ as a Gelfand-Tsetlin triangle whose $i$ th row $(1 \leq i \leq n)$ lists, in decreasing order (with 0's appended or deleted from the end as needed), the number of parts less than or equal to $n-i+1$ in the successive rows of the tableau:

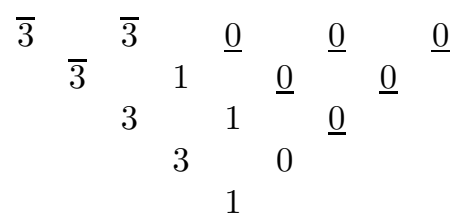

This tableau splits into three parts: a triangle of $B$ 's (overlined, with top row of length $A$ ), a triangle of 0 's (underlined, with top row of length $n-A$ ), and an $(n-A)$-by- $(A)$ rectangle. If we flip this rectangle across the line $x+y=0$, so that the top corner becomes the left corner and vice versa, we get a $P$-array with entries between 0 and $B$ :

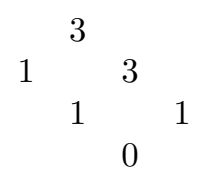

If we divide each entry by $B$, we get a point $v(T)$ in $\mathcal{O}(P)$ from which one can recover $T$ by reversing all the above steps. One can show that $v\left(\pi_{\mathcal{S}}(T)\right)=\pi_{\mathcal{P}}(v(T))$. Indeed, the file-toggle operations (in which one performs piecewise-linear toggling at all $x \in P$ belonging to the $i$ th file of $[n-A] \times[A]$, with $1 \leq i \leq n$; see 6) can be shown to correspond respectively to the $n$ Bender-Knuth involutions on the Young tableau, whose composition gives $\pi_{\mathcal{S}}$.

The vertices of $\mathcal{O}(P)$ correspond to the 0,1 -valued functions $f$ on $P$ with the property that $x \leq y$ in $P$ implies $f(x) \leq f(y)$ in $\{0,1\}$; these are precisely the indicator functions of filters. Filters are in bijection with order ideals by way of the complementation map, so the vertices of $\mathcal{O}(P)$ are in bijection with the elements of the lattice $J(P)$. Each toggle operation acts as a permutation on the vertices of $\mathcal{O}(P)$. Indeed, if we think of each vertex of $\mathcal{O}(P)$ as determining a cut of the poset $P$ into an upset (filter) $S_{\text {up }}$ and a complementary downset (order ideal) $S_{\text {down }}$ (the pre-image of 1 and 0 , respectively, under the order-preserving map from $P$ to $\{0,1\})$, then the effect of the toggle operation $\tau_{x}(x \in P)$ is just to move $x$ from $S_{\text {up }}$ to $S_{\text {down }}$ (if $x$ is in $S_{\text {up }}$ ) or from $S_{\text {down }}$ to $S_{\text {up }}$ (if $x$ is in $S_{\text {down }}$ ) unless this would violate the property that $S_{\text {up }}$ must remain an upset and $S_{\text {down }}$ must remain a downset. In particular, we can see that when our point $v \in \mathcal{O}(P)$ is a vertex associated with the cut $\left(S_{\text {up }}, S_{\text {down }}\right)$, the effect of $\tau_{x}$ on $S_{\text {down }}$ is just toggling the order ideal $S_{\text {down }}$ at the element $x \in P$.

Cameron and Fon-Der-Flaass (1995) showed that rowmotion acting on $J([a] \times[b])$ is of order $a+b$. (Subsequently Striker and Williams (2012) gave a simpler proof, by showing that promotion is of order $a+b$ and that rowmotion is conjugate to promotion.) The same is true of piecewise-linear rowmotion and promotion acting on $\mathcal{O}([a] \times[b])$ :

Theorem 1 For $P=[a] \times[b]$, the maps $\rho_{\mathcal{P}}$ and $\pi_{\mathcal{P}}$ are of order $a+b$.

It seems plausible that one might be able to deduce the order of $\rho_{\mathcal{P}}$ and $\pi_{\mathcal{P}}$ from the order of $\rho$ and $\pi$, but we have not been able to find such an argument. ${ }^{\text {(iii) }}$ Instead, our proof of Theorem 1 detours through the notions of birational promotion and rowmotion.

\footnotetext{
(iii) The Coxeter hyperplane arrangement of type $A$ divides the order polytope into simplices, and on each simplex the maps $\rho_{\mathcal{P}}$ and $\pi_{\mathcal{P}}$ are not just piecewise-linear but actually linear (by which we really mean "affine"), and one might hope to base a proof of
} 


\section{Birational toggling}

The definition of the piecewise-linear toggling operation via formulas (1)-(3) involves only addition, subtraction, min, and max. Consequently one can define birational transformations on $\left(\mathbb{R}^{+}\right)^{P}$ with formal resemblance to the toggle operations on $\mathcal{O}(P)$. This transfer makes use of a dictionary in which 0 , addition, subtraction, max, and min are respectively replaced by 1 , multiplication, division, addition, and parallel addition (defined below), resulting in a subtraction-free rational expression. ${ }^{\text {(iv) }}$ Parallel addition can be expressed in terms of the other operations, but taking a symmetrical view of the two forms of addition turns out to be fruitful. Indeed, in setting up the correspondence we have a choice to make: by "series-parallel duality", one could equally well use a dictionary that switches the roles of addition and parallel addition. We hope the choice that we have made here will prove to be convenient.

For $x, y$ satisfying $x+y \neq 0$, we define the parallel sum of $x$ and $y$ as $x \| y=x y /(x+y)$. In the case where $x, y$ and $x+y$ are all nonzero, $x y /(x+y)$ is equal to $1 /\left(\frac{1}{x}+\frac{1}{y}\right)$, which clarifies the choice of notation and terminology: if two electrical resistors of resistance $x$ and $y$ are connected in parallel, the compound circuit has an effective resistance of $x \| y$. If $x$ and $y$ are in $\mathbb{R}^{+}$, then $x+y$ and $x \| y$ are in $\mathbb{R}^{+}$as well. Also, $\|$is commutative and associative, so that a compound parallel sum $x\|y\| z \| \cdots$ is well-defined; it equals the product $x y z \cdots$ divided by the sum of all products that omit exactly one of the variables, and in the case where $x, y, z, \ldots$ are all positive, it can also be written as $1 /\left(\frac{1}{x}+\frac{1}{y}+\frac{1}{z}+\cdots\right)$.

Given a non-empty set $S=\left\{s_{1}, s_{2}, \ldots\right\}$, let $\sum^{+} S$ denote $s_{1}+s_{2}+\cdots$ and $\sum^{\|} S$ denote $s_{1}\left\|s_{2}\right\| \cdots$. Then for $v=\left(v_{0}, v_{1}, \ldots, v_{p}, v_{p+1}\right) \in\left(\mathbb{R}^{+}\right)^{p+2}$ with $v_{0}=v_{p+1}=1$ and for $1 \leq i \leq p$ we define

$$
\tau_{i}(v)=\left(v_{0}, v_{1}, \ldots, v_{i-1}, L R / v_{i}, v_{i+1}, \ldots, v_{p}, v_{p+1}\right),
$$

with

$$
L=\sum^{+}\left\{v_{j}: 0 \leq j \leq p+1, x_{j} \lessdot x_{i}\right\}
$$

and

$$
R=\sum^{\|}\left\{v_{j}: 0 \leq j \leq p+1, x_{j} \gg x_{i}\right\} .
$$

We call the maps $\tau_{i}:\left(\mathbb{R}^{+}\right)^{P} \rightarrow\left(\mathbb{R}^{+}\right)^{P}$ given by (4)-(6) birational toggle operations, as opposed to the piecewise-linear toggle operations treated in the previous section. ${ }^{(v)}$ As the 0 th and $p+1$ st coordinates of $v$ are not affected by any of the toggle operations, we can just omit those coordinates, reducing our toggle operations to actions on $\left(\mathbb{R}^{+}\right)^{p}$. Since $L R /\left(L R / v_{i}\right)=v_{i}$, each birational toggle operation is an involution on the orthant $\left(\mathbb{R}^{+}\right)^{p}$. As in the preceding section, we identify $\left(\mathbb{R}^{+}\right)^{p}$ with $\left(\mathbb{R}^{+}\right)^{P}$. The birational toggle operations are analogous to the piecewise-linear toggle operations (in a sense to be made precise below), so it is natural to define birational rowmotion $\rho_{\mathcal{B}}:\left(\mathbb{R}^{+}\right)^{P} \rightarrow\left(\mathbb{R}^{+}\right)^{P}$ as the composite operation accomplished by toggling from top to bottom, and to define birational promotion $\pi_{\mathcal{B}}:\left(\mathbb{R}^{+}\right)^{P} \rightarrow\left(\mathbb{R}^{+}\right)^{P}$ as the composite operation accomplished by toggling from left to right.

Continuing our running example $P=[2] \times[2]=\{w, x, y, z\}$, let $v=(1,2,3,4) \in \mathbb{R}^{P}$, corresponding to the positive function $f$ that maps $w, x, y, z$ to $1,2,3,4$, respectively, with $f(\widehat{0})=f(\widehat{1})=1$. Under

\footnotetext{
Theorem 1 on this; unfortunately, the images of these simplices under $\rho_{\mathcal{P}}$ and $\pi_{\mathcal{P}}$ are not themselves simplices in this dissection, so the most simple sort of proof one might imagine does not work.

(iv) The authors are indebted to Arkady Berenstein for pointing out the details of this transfer of structure from the piecewise-linear setting to the birational setting.

(v) In principle we should use a different symbol than $\tau_{i}$, but in practice it should always be clear whether we are referring to piecewise-linear operations or birational operations.
} 
the action of $\tau_{z}, \tau_{y}, \tau_{x}$, and $\tau_{w}$, the vector $v=(1,2,3,4)$ gets successively mapped to $\left(1,2,3, \frac{5}{4}\right)$, $\left(1,2, \frac{5}{12}, \frac{5}{4}\right),\left(1, \frac{5}{8}, \frac{5}{12}, \frac{5}{4}\right)$, and $\left(\frac{1}{4}, \frac{5}{8}, \frac{5}{12}, \frac{5}{4}\right)=\rho_{\mathcal{B}}(v)$.

For simplicity, we have defined $\pi_{\mathcal{B}}$ as a map from $\left(\mathbb{R}^{+}\right)^{P}$ to itself. However, $\pi_{\mathcal{B}}$ can be extended to a map from a dense open subset of $\mathbb{R}^{P}$ to itself, and indeed, from a dense open subset $U$ of $\mathbb{C}^{P}$ to itself. All expressions we consider are well-defined on the open orthant $\left(\mathbb{R}^{+}\right)^{P}$, and all the theorems we prove amount to identities that are valid when all variables lie in this orthant; this implies that the identities hold outside of some singular variety in $\mathbb{C}^{P}$. Identifying the singular subvariety on which $\pi_{\mathcal{B}}$ (or one of its powers) is undefined seems like an interesting question, but it is one that we leave to others. Alternatively, Tom Roby has pointed out that one can replace $\mathbb{R}^{+}$by a ring of rational functions in formal indeterminates indexed by the elements of $P$, thereby avoiding the singularity issue (once one checks that the rational functions in question can be expressed as ratios of polynomials with positive coefficients).

Piecewise-linear rowmotion and promotion can be viewed as tropicalizations of birational rowmotion and promotion. To the extent that facts about birational toggling can be formulated as (complicated but finite) identities in subtraction-free arithmetic, the dictionary alluded to at the start of section 3 allows one to carry the identities to the "max, min, plus" setting. ${ }^{(v i)}$ For instance, when in a later section we prove that $\rho_{\mathcal{B}}^{n}$ and $\pi_{\mathcal{B}}^{n}$ act trivially on $\left(\mathbb{R}^{+}\right)^{P}$ (with $P=[a] \times[b]$ and $n=a+b$ ), it will follow immediately that $\rho_{\mathcal{P}}^{n}$ and $\pi_{\mathcal{P}}^{n}$ act trivially on $\mathbb{R}^{P}$. (Here we gloss over the role that $\widehat{0}$ and $\widehat{1}$ play. Our treatment of birational toggling assumes $\widehat{f}(\widehat{0})=\widehat{f}(\widehat{1})=1$ but our treatment of piecewise-linear toggling assumes $\widehat{f}(\widehat{0})=0 \neq 1=\widehat{f}(\widehat{1})$. The full version of the paper addresses this issue with an appropriate dehomogenization lemma.)

\section{Birational rowmotion and Stanley's transfer map}

Although most of our work with rowmotion treats it as a composition of $|P|$ toggles (from the top to the bottom of $P$ ), we noted in section 1 that $\rho$ can also be defined as a composition of three operations $\alpha_{1}$, $\alpha_{2}, \alpha_{3}$. ${ }^{\text {(vii) }}$ This alternative definition can be lifted to the piecewise-linear and birational settings.

For the piecewise-linear setting, we first recall the definition of the chain polytope $\mathcal{C}(P)$ of a poset $P$ as defined by Stanley (1986). A chain in a poset $P$ is a totally ordered subset of $P$, and a maximal chain in a poset $P$ is a chain that is not a proper subset of any other chain. If the poset $P$ is ranked, with all maximal elements having the same rank, then the maximal chains in $P$ are precisely those chains that contain an element of every rank. The chain polytope of a poset $P$ is the set of maps from $P$ to $[0,1]$ such that for every chain $C$ in $P$ (or, equivalently, for every maximal chain $C$ in $P$ ),

$$
\sum_{x \in C} f(x) \leq 1
$$

Just as the vertices of the order polytope of $P$ correspond to the indicator functions of the filters of $P$, the vertices of the chain polytope of $P$ correspond $^{\text {(viii) }}$ to the indicator functions of the antichains of $P$.

Stanley defines the transfer map $\Phi: \mathcal{O}(P) \rightarrow \mathbb{R}^{P}$ via the formula

$$
(\Phi f)(x)=\min \{f(x)-f(y): y \in \widehat{P}, x \gg y\}
$$

(vi) We are indebted to Colin McQuillan and Will Sawin for clarifying this point; see MathOverflow (2013).

(vii) Indeed this was the way in which Brouwer and Schrijver originally defined their operation $F$, in the context of the Boolean lattices [2] $\times[2] \times \cdots \times[2]$.

(viii) One direction of this claim is easy: since every antichain intersects every chain of $P$ in at most one element of $P$, the indicator function of an antichain must correspond to a point in $\mathcal{C}(P)$. For the other direction, see Theorem 2.2 of Stanley (1986). 
for all $x \in P$ (recall that we have $f(\widehat{0})=0$ ). Stanley proves that $\Phi$ is a bijection between $\mathcal{O}(P)$ and $\mathcal{C}(P)$ that carries the vertices of the former to the vertices of the latter. The inverse of $\Phi$ is given by ${ }^{(i x)}$

$$
(\Psi g)(x)=\max \left\{g\left(y_{1}\right)+g\left(y_{2}\right)+\cdots+g\left(y_{k}\right): \widehat{0} \lessdot y_{1} \lessdot y_{2} \lessdot \cdots \lessdot y_{k}=x\right\} .
$$

Let $\tilde{\mathcal{O}}(P)$ be the set of order-reversing maps from $P$ to $[0,1]$. We now define bijections $\alpha_{1}: \tilde{\mathcal{O}}(P) \rightarrow$ $\mathcal{O}(P), \alpha_{2}: \mathcal{O}(P) \rightarrow \mathcal{C}(P)$, and $\alpha_{3}: \mathcal{C}(P) \rightarrow \tilde{\mathcal{O}}(P)$ given by the following recipes:

(1) for $f \in \tilde{\mathcal{O}}(P)$, let $\alpha_{1}(f)$ be defined by

$$
\left(\alpha_{1}(f)\right)(x)=1-f(x) ;
$$

(2) for $f \in \mathcal{O}(P)$, let $\alpha_{2}(f)$ be defined by

$$
\left(\alpha_{2} f\right)(x)=\min \{f(x)-f(y): y \in \widehat{P}, x>y\} ;
$$

and

(3) for $f \in \mathcal{C}(P)$, let $\alpha_{3}(f)$ be defined by

$$
\left(\alpha_{3} f\right)(x)=\max \left\{f\left(y_{1}\right)+f\left(y_{2}\right)+\cdots+f\left(y_{k}\right): x=y_{1} \lessdot y_{2} \lessdot \cdots \lessdot y_{k} \lessdot \widehat{1}\right\} .
$$

Note that $\alpha_{2}$ is $\Phi$ and that $\alpha_{3}$ is $\Psi$ (aka $\Phi^{-1}$ ) "turned upside down". It is not hard to check that (10) can be replaced by the recursive definition

$$
\left(\alpha_{3} f\right)(x)=f(x)+\max \left\{\left(\alpha_{3} f\right)(y): y \in \widehat{P}, y>x\right\}
$$

which turns out to be the form most suitable for lifting to the birational setting.

Theorem $2 \rho_{\mathcal{P}}=\alpha_{1} \circ \alpha_{3} \circ \alpha_{2}$.

(Note that $\alpha_{1} \circ \rho_{\mathcal{P}} \circ \alpha_{1}=\alpha_{3} \circ \alpha_{2} \circ \alpha_{1}$, as in the original definition of $\rho$.)

Similarly, in the birational setting put

$$
\begin{aligned}
& \left(\alpha_{1} f\right)(x)=1 / f(x), \\
& \left(\alpha_{2} f\right)(x)=\sum^{\|}\left\{f(x) / f(y): y \in x^{-}\right\}, \text {and } \\
& \left(\alpha_{3} f\right)(x)=f(x) \sum^{+}\left\{\left(\alpha_{3} f\right)(y): y \in x^{+}\right\},
\end{aligned}
$$

where $x^{+}$denotes $\{y \in \widehat{P}: y \gg x\}$ and $x^{-}$denotes $\{y \in \widehat{P}: x>y\}$. (Note that definition (14), like definition (11), is recursive.)

Theorem $3 \rho_{\mathcal{B}}=\alpha_{1} \circ \alpha_{3} \circ \alpha_{2}$.

Of course the $\alpha$ 's in Theorem 3 are not the $\alpha$ 's in Theorem 2 but their birational counterparts.

In the full paper, we derive Theorem 2 from Theorem 3 by tropicalization and dehomogenization.

(ix) This is not precisely the definition of $\Psi$ that Stanley gives, but the two definitions are easily seen to be equivalent. 


\section{Recombination and Reciprocal Symmetry}

As was noted by Striker and Williams (2012), there is an intimate relationship between rowmotion and promotion in rc-embedded posets: the two maps have the same orbit structure because they are conjugate as elements of the toggle group. This relationship becomes even clearer in the piecewise-linear and birational settings. Let $P=[2] \times[2]$. Here is the $\rho_{\mathcal{B}}$-orbit of $(1,2,3,4)$ :

\begin{tabular}{|c|c|c|c|}
\hline 1 & 2 & 3 & 4 \\
\hline $1 / 4$ &,$\quad 5 / 8$ & $5 / 12$ & $5 / 4$ \\
\hline $4 / 5$ &,$\quad 1 / 3$ & $1 / 2$ & $5 / 6$ \\
\hline $6 / 5$ &,$\quad 12 / 5$ & $8 / 5$ & 1 \\
\hline
\end{tabular}

Here is the $\pi_{\mathcal{B}}$-orbit of $(1,2,5 / 12,5 / 4)$ :

$\left.\begin{array}{cccccccc}(1 & , & 2 & , & 5 / 12 & , & 5 / 4 & \\ (1 / 4 & , & 5 / 8 & , & 1 / 2 & , & 5 / 6 & ) \\ (4 / 5 & , & 1 / 3 & , & 8 / 5 & , & 1 & ) \\ (6 / 5 & , & 12 / 5 & , & 3 & , & 4\end{array}\right)$

Note that the same numbers appear as entries in both orbits, with the same multiplicity. More specifically, given $P=[a] \times[b]$, define the recombination map $D$ as the map from the set of $P$-arrays to itself such that for every $P$-array $f$, the $(i, j)$ entry in $D(f)$ is the $(i, j)$ entry in $\rho_{\mathcal{B}}^{i-1}(f)$.

Theorem 4 (the "recombination lemma"): $D \circ \pi_{\mathcal{B}}=\rho_{\mathcal{B}} \circ D$.

It follows from Theorem 4 that $D$ is invertible and that $\pi_{\mathcal{B}}$ and $\rho_{\mathcal{B}}$ have the same orbit-structure.

A seemingly much deeper fact is the following consequence of the work of Grinberg and Roby (2014) (Theorem 10.6 in particular).

Theorem 5 (reciprocal symmetry): The $(a-i+1, b-j+1)$ entry in $\rho_{\mathcal{B}}^{a+b+1-i-j}(f)$ is the reciprocal of the $(i, j)$ entry in $f$.

Applying this theorem twice yields the conclusion that for $n=(a+b+1-i-j)+(a+b+1-(a-$ $i+1)-(b-j+1))=a+b$, the $(i, j)$ entry in $\rho_{\mathcal{B}}^{n}$ is the reciprocal of the reciprocal of the $(i, j)$ entry in $f$. This implies that $\rho_{\mathcal{B}}^{n}$ is the identity map (and recombination then assures us that that $\pi_{\mathcal{B}}^{n}$ is the identity map as well). The fact that $\rho^{n}$ acts trivially on $J([a] \times[b])$ was first proved by Fon-Der-Flaass (1993).

These facts have implications in the piecewise-linear setting. The recombination property says that the $(i, j)$ entry in $D(f)$ is the $(i, j)$ entry in $\rho_{\mathcal{B}}^{i-1}(f)$, and reciprocal symmetry says that the $(a-i+1, b-j+1)$ entry in $\rho_{\mathcal{P}}^{a+b+1-i-j}(f)$ is 1 minus the $(i, j)$ entry in $f$. We also may conclude that $\rho_{\mathcal{P}}^{n}$ and $\pi_{\mathcal{P}}^{n}$ are the identity map. The last of these conclusions, in combination with our remarks in section 2 linking certian $P$-arrays with semistandard Young tableaux, gives us a new proof of the standard fact that Schützenberger promotion on standard tableaux of fixed rectangular shape with entries bounded by $n$ has order $n$.

We stress that recombination is not specific to $[a] \times[b]$, but applies to any rc-embedded poset, even in cases where rowmotion is not of finite order. The recombination lemma is heavily based on Theorem 5.4 in Striker and Williams (2012) (construction of an equivariant bijection). 


\section{File-toggling and promotion}

Here we restrict to $P$ of the form $[a] \times[b]$, with $n=a+b$. The birational toggle operations $\tau_{i}$, combined in unconstrained fashion, generate a group that is infinite when $a>1$ or $b>1$ (we prove this in detail in the full article for the case $a=b=2$ ), and its structure is likely to be quite complicated, but some of the subgroups admit homomorphisms to the symmetric group $S_{n}$, and they can be useful for understanding rowmotion and promotion. One such subgroup, generated by $n-1$ involutions associated with the respective ranks of $P$, was discovered by Grinberg and Roby (2014). Here we study a different subgroup, generated by $n-1$ involutions associated with the respective files of $P$.

Recall that $[a] \times[b]$ can be partitioned into files numbered 1 through $n-1$ from left to right. Given $f: \widehat{P} \rightarrow \mathbb{R}^{+}$with $f(\widehat{0})=f(\widehat{1})=1$, let $p_{i}(1 \leq i \leq n-1)$ be the product of the numbers $f(x)$ with $x$ belonging to the $i$ th file of $P$, let $p_{0}=p_{n}=1$, and for $1 \leq i \leq n$ let $q_{i}=p_{i} / p_{i-1}$. Call $q_{1}, \ldots, q_{n}$ the quotient sequence associated with $f$, and denote it by $Q(f)$. This is analogous to the difference sequence introduced in Propp and Roby (2013). Note that the product $q_{1} \cdots q_{n}$ telescopes to $p_{n} / p_{0}=1$. For $i$ between 1 and $n-1$, let $\tau_{i}^{*}$ be the product of the commuting involutions $\tau_{x}$ for all $x$ belonging to the $i$ th file. Lastly, given a sequence of $n$ numbers $w=\left(w_{1}, \ldots, w_{n}\right)$, and given $1 \leq i \leq n-1$, define $\sigma_{i}(w)=\left(w_{1}, \ldots, w_{i-1}, w_{i+1}, w_{i}, w_{i+2}, \ldots, w_{n}\right)$; that is, $\sigma_{i}$ switches the $i$ th and $i+1$ st entries of $w$.

Lemma 6 For all $1 \leq i \leq n-1$, and for all $f$,

$$
Q\left(\tau_{i}^{*} f\right)=\sigma_{i} Q(f)
$$

That is, toggling the ith file of $f$ swaps the ith and $i+1$ st entries of the quotient sequence of $f$.

Recalling that $\pi_{\mathcal{B}}$ is the composition $\tau_{n-1}^{*} \circ \cdots \circ \tau_{1}^{*}$, we have:

Corollary $7 Q\left(\pi_{\mathcal{B}} f\right)$ is the leftward cyclic shift of $Q(f)$.

\section{Homomesy}

Given a set $X$, an operation $T: X \rightarrow X$ whose $n$th power is the identity map on $X$, and a function $F$ from $X$ to a field $\mathcal{K}$ of characteristic 0 , we say that $F$ is homomesic relative to (or under the action of) $T$, or that the triple $(X, T, F)$ exhibits homomesy, if for all $x \in X$ the average

$$
\frac{1}{n} \sum_{k=0}^{n-1} F\left(T^{k}(x)\right)
$$

equals some $c$ independent of $x$. We also say in this situation that the function $F$ (which we will sometimes call a functional on $X$ ) is c-mesic relative to the map T. The article by Propp and Roby (2013) gives examples of combinatorial situations in which homomesy holds. See also Bloom et al. (2013).

Theorem 5 yields as a corollary that $\left(\left(\mathbb{R}^{+}\right)^{P}, \rho_{\mathcal{B}}, F\right)$ is 0 -mesic, where $F(f)=\log (f(i, j) f(a+1-$ $i, b+1-j)$ ) (factors cancel in pairs). Applying recombination, we see that the same is true if rowmotion is replaced by promotion. In both cases, tropicalizing yields homomesy for $F(f)=f(i, j)+f(a+1-$ $i, b+1-j$ ) under piecewise-linear rowmotion and promotion.

A different sort of homomesy comes from the files of $[a] \times[b]$. Using Corollary 7 , one can show that for each $i$ between 1 and $n-1$, if one defines $F_{i}(f)$ as the logarithm of the product of the values of 
$f(x)$ as $x$ ranges over the $i$ th file of $[a] \times[b]$, then $\left(\left(\mathbb{R}^{+}\right)^{P}, \rho_{\mathcal{B}}, F_{i}\right)$ is 0 -mesic. This can be carried to the piecewise-linear setting as well. Restricting to the vertices of $\mathcal{O}(P)$, one obtains the main homomesy theorem of Propp and Roby (2013).

We can see both forms of homomesy on display in the rowmotion orbit shown at the start of section 5 . For instance, the middle file of the poset consists of the elements $w$ and $z$, associated with the entries $v_{1}$ and $v_{4}$ of each vector $v$. Defining $F(f)$ as $\log f(w) f(z)$, we see that over the orbit the function $F$ takes on the values $\log 4, \log 5 / 16, \log 2 / 3$, and $\log 6 / 5$, which sum to 0 .

Theorem 8 Given $P=[a] \times[b]$, with $n=a+b$, define functionals $F_{i, j}(1 \leq i \leq a, 1 \leq j \leq b)$ and $F_{k}$ $(1 \leq k \leq n-1)$ by

$$
\begin{gathered}
\left.F_{i, j}(f)=f(i, j)+f(a+1-i, b+1-j)\right), \\
F_{k}(f)=\sum_{j-i=k-a} f(i, j) .
\end{gathered}
$$

These functionals are all homomesic under the action of $\rho_{\mathcal{P}}$ and $\pi_{\mathcal{P}}$.

Theorem 9 Given $P=[a] \times[b]$, with $n=a+b$, define functionals $F_{i, j}(1 \leq i \leq a, 1 \leq j \leq b)$ and $F_{k}$ $(1 \leq k \leq n-1)$ by

$$
\begin{gathered}
F_{i, j}(f)=\log (f(i, j) f(a+1-i, b+1-j)), \\
F_{k}(f)=\log \left(\prod_{j-i=k-a} f(i, j)\right) .
\end{gathered}
$$

These functionals are all homomesic under the action of $\rho_{\mathcal{B}}$ and $\pi_{\mathcal{B}}$.

The recombination lemma easily implies that a functional $F$ is homomesic under rowmotion if and only if it is homomesic under promotion. Also, any linear combination of homomesic functions is homomesic.

In the full version of the article, a kind of converse of Theorem 8 will be proved:

Theorem 10 Given $P=[a] \times[b]$, with $p=a b$, let $F$ be some function in the span of the $p$ evaluation functions $f \mapsto f(i, j)$ (with $1 \leq i \leq a, 1 \leq j \leq b$ ), such that $F$ is homomesic under the action of $\rho_{\mathcal{P}}$ (or equivalently under the action of $\left.\pi_{\mathcal{P}}\right)$; then $F$ must be a linear combination of the functional $F_{i, j}$ and $F_{k}$ defined in Theorem 8.

Let $V$ be the vector space spanned by the functionals $F_{i, j}$ and $F_{k}$. It should be noted that the functionals $F_{i, j}$ and $F_{k}$ have linear dependencies, so although they span $V$, they are not a basis of $V$.

Although we have restricted ourselves to $\left(\mathbb{R}^{+}\right)^{P}$ for simplicity, to the extent that our main results are complicated but finite subtraction-free identities, results like these homomesy theorems, or the fact that rowmotion and promotion are of order $n$, apply throughout the complement of some proper subvariety of $\mathbb{C}^{P}$ (though we need to use $\log |z|$ in place of $\log z$ ). Also note that our birational maps are homogeneous, so projective counterparts of rowmotion and promotion can be defined and are likely to be helpful.

\section{Acknowledgements}

This work was supported by a grant from NSF. The authors are grateful to Arkady Berenstein, Darij Grinberg, Alex Postnikov, Tom Roby, Richard Stanley, and Jessica Striker for helpful conversations, and to the referees for helpful suggestions. 


\section{References}

J. Bloom, O. Pechenik, and D. Saracino. A homomesy conjecture of J. Propp and T. Roby. 2013. Preprint, 2013: arXiv:1308.0546.

A. Brouwer and A. Schrijver. On the period of an operator, defined on antichains. Math Centrum report ZW, 24/74, 1974.

P. Cameron and D. Fon-Der-Flaass. Orbits of antichains revisited. European J. Combin., 16(6):545-554, 1995. ISSN 0195-6698.

D. Einstein and J. Propp. Combinatorial, piecewise-linear, and birational homomesy for products of two chains. 2014. Preprint, 2014; arXiv:1310.5294.

D. Fon-Der-Flaass. Orbits of antichains in ranked posets. European J. Combin., 14(1):17-22, 1993. ISSN 0195-6698.

D. Grinberg and T. Roby. Iterative properties of birational rowmotion. 2014. Preprint, 2014: arXiv:1402.6178.

A. Kirillov and A. Berenstein. Groups generated by involutions, Gelfand-Tsetlin patterns and combinatorics of Young tableaux. Algebra i Analiz, 7(1):92-152, 1995. http://pages . uoregon. edu/ arkadiy/bk1.pdf.

MathOverflow, 2013. http://mathoverflow. net/questions/127108/ do-all-subtraction-free-identities-tropicalize.

J. Propp and T. Roby. Homomesy in products of two chains. DMTCS Proceedings, pages 945-956, 2013. ISSN 1365-8050. FPSAC 2013, http://www.dmtcs.org/dmtcs-ojs/index.php/ proceedings/article/view/dmAs0180/4290.

M.-P. Schützenberger. Promotion des morphismes d'ensembles ordonnés. Discrete Mathematics, 2(1): 73-94, 1972.

R. Stanley. Two poset polytopes. Discrete and Computational Geometry, 1:9-23, 1986.

R. P. Stanley. Promotion and evacuation. Electronic Journal of Combinatorics, 16(2):R9, 2009.

R. P. Stanley. Enumerative Combinatorics, Vol. 1, volume 49. Cambridge University Press, 2011.

J. Striker and N. Williams. Promotion and rowmotion. European Journal of Combinatorics, 33(8):19191942, 2012.

M. van Leeuwen. The Robinson-Schensted and Schützenberger algorithms, an elementary approach. Electronic Journal of Combinatorics, 3(2):R15, 1996. 